ORIGINAL RESEARCH ARTICLE

\title{
Culture of C6 glioma cells and establishment of model
}

\author{
Shumin Liang, Yijun Chen,Kaifei Wang \\ Medical College, Weifang University of Technology, Shandong, China
}

\begin{abstract}
Objectives: To establish a simple, reliable, stable rat $\mathrm{C} 6$ glioma model, and to provide an operating platform for studying the pathogenesis and prevention and treatment of glioma. Methods: $\mathrm{C} 6$ rat glioma cells were cultured in vitro and cultured in the right caudate putamen of SD rats. After implantation, the survival status of rats was observed and the pathological sections were made. HE is dyeing under light microscope. Results: After 6 days of inoculation of C6 glioma cells, the survival status was good at about 7 days, and the intracranial hypertension was more obvious at about 14 days. Most of them were in the endangered state. In the 18 specimens of the rats, except for the accidental death, the other tumor formation rate was $75 \%$. The tumor structure was obviously displaced with the prolongation of the age of the tumor. HE staining was used to observe the formation of glioma in rat brain. Conclusion: The animal model of C6 glioma is reliable and stable. Its tumor growth characteristics and pathological features are similar to those of human glioma, which can be used as an ideal model for clinical study of glioma.

KEYWORDS: glioma; stereotactic technique; animal model; pathology
\end{abstract}

\section{Introduction}

\subsection{Research background}

Brain glioma is a class of common human tumors that endanger human health. Regardless of surgery, radiotherapy, chemotherapy, hyperthermia or guided therapy, the effect is not good, the average survival time of only 8 months. Although the overall effect of treatment has improved, but the prognosis is still poor. In order to find a more effective therapy, the establishment of brain glioma animal model for experimental treatment, especially experimental gene therapy to provide effective means of research.

\subsection{Research status}

The method of establishing glioma is three types: induced type, transplantation type and transgenic genotype. The first two methods are used for a long time, and the animal model obtained is currently widely used. In contrast, the transgenic animal model has the advantages of molecular mechanism clear, the establishment of system stability and good repeatability. The disadvantages are tissue-specific injection is relatively difficult and the model is difficult to build. The main advantages of the transplant model of animal brain tumor are the transplanted tumor strain is easy to survive, easy to establish and preserve, and retain the good histopathology, molecular biology and cell biology of human glioma, tumor release / chemotherapy and other good models. There are similar allograft models and xenograft models. C6 glioma cell line in 1968 by Benda et al. from the rat brain glioma isolated and cloned to establish, with glioma of the typical biological characteristics, can be a good simulation of human glioma pathophysiological changes and clinical processes. At present, mainly through the subculture to study the characteristics of the various stages of growth.

\subsection{The purpose of this study}

According to the related literature, we try to establish a simple, reliable and stable rat model of glioma, and provide an operating platform for studying the pathogenesis and treatment of glioma. Similar to the pathological features of human glioma, it can be used as an ideal model for clinical study of clinical glioma.

Copyright (C) 2017 -. This is an Open Access article distributed under the terms of the Creative Commons Attribution-NonCommercial 4.0 International License (http://creativecommons.org/licenses/by-nc/4.0/), permitting all non-commercial use, distribution, and reproduction in any medium, provided the original work is properly cited. 


\section{Experimental materials}

\subsection{Cell lines and experimental animals}

\subsubsection{Cell lines}

C6 glioma cell line provided by the Chinese Academy of Sciences Shanghai cells.

\subsubsection{Experimental animals}

SD rats, male, weight 180 - 220g, provided by the Experimental Animal Center of Anhui Medical University.

\subsection{2 Instruments and reagents}

\subsubsection{Instrument}

MAL175 type carbon dioxide incubator (Sanyo, Japan), ultra-clean table (Hal-East Electronic Technology Development Co., Ltd.), brain stereotactic instrument (Shenzhen Ruiwo De Life Technology Co., Ltd.), desktop refrigerated centrifuge Beckman, CS-15R), inverted microscope (Japan Olym-pus), $25 \mu \mathrm{L}$ microinjector (Q / AMGK011998, Shanghai high-tech glass instrument factory), 3.0T nuclear magnetic resonance instrument, rat special coil purchased from the United States GE company. Conventional surgical instruments were used.

\subsubsection{Reagents}

DMEM (dulbecco modified eagle med -ium) medium;

Newborn calf serum is GIBCO products, agar is produced by Sigma;

Gd-DTPA is a product of Beijing Hokuriku Pharmaceutical Co., Ltd.;

GFAP protein antiserum and SAB kit purchased in Wuhan Boster Company;

Streptomycin, antibiotics, PBS (Shanghai Biyun days Biotechnology Co., Ltd.).

\section{3. Experimental methods}

\subsection{1 C6 glioma cell culture}

\subsubsection{Cell resuscitation}

Liquid nitrogen cans in the ampoule, quickly into the $37{ }^{\circ} \mathrm{C}$ water bath, and from time to time to shake, in 1 minute to make it completely melt. Sterile cells were removed. Frozen tube with $75 \%$ alcohol wipe disinfection, open the lid, with a straw to the cell suspension into the centrifuge tube, and then dropping $10 \mathrm{ml}$ of culture medium. Centrifuge for 10 minutes at $800 \mathrm{r} / \mathrm{min}$, discard the supernatant solution, add the appropriate amount of culture medium after inoculation in the culture bottle, inoculation concentration $1 \times 107 / \mathrm{L}$, set $37^{\circ} \mathrm{C}$ incubator culture, the next day to replace the culture medium, Continue to cultivate and observe the growth. If the cell density is high, timely passage. The amount of culture medium added depends on the number of cells to be frozen, and if the amount of cryopreservation is 106, the cell is diluted 10 times to 105 . Figure:

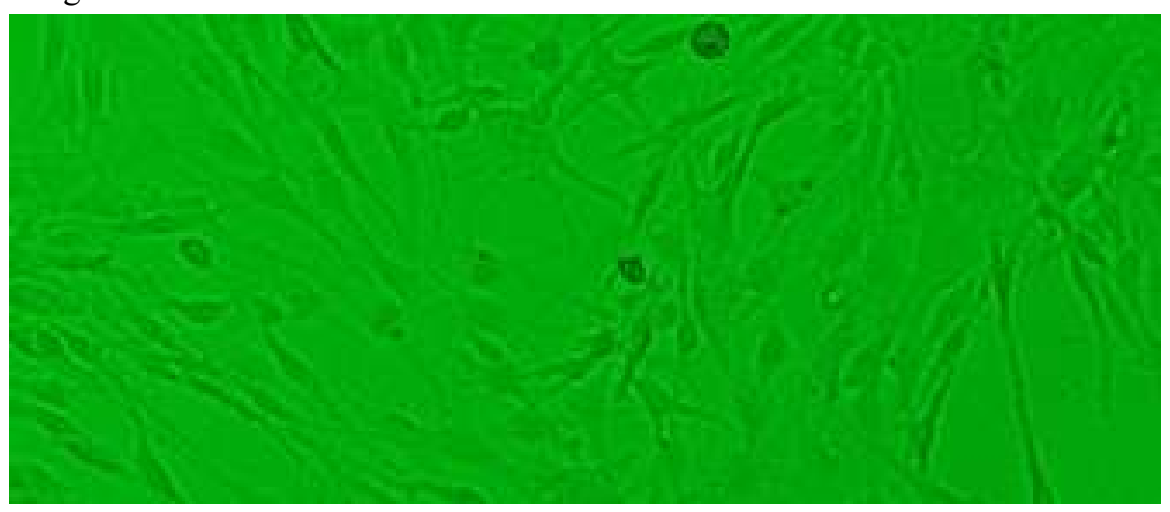




\subsubsection{Cell passage}

1. The medium was aspirated and the cells were washed twice with $2 \mathrm{ml}$ of PBS;

2. Add $1 \mathrm{ml}$ trypsin digestion, room temperature for 30 s or so, gently blow the cells with a gun;

3. Under the microscope, if the cells appear obvious contraction, from the culture dish; and visual observation of the bottom of the cell culture found that the cell morphology changes significantly;

4. At this time by adding serum containing complete cell culture, continue to blow cells;

5. Transfer the culture medium in the culture dish, add fresh culture medium, into the incubator culture.

\subsection{2 Establishment of C6 Stereotaxic C6 Cell Establishment Model}

\subsubsection{Preparation of injection cells}

C6 cells were cultured in DMEM medium containing 10\% neonatal calf serum, penicillin $100 \mathrm{U} / \mathrm{mL}$ and streptomycin $100 \mu \mathrm{g} / \mathrm{mL}$ in $5 \% \mathrm{CO} 2$ at 37 . Before the inoculation, the logarithmic growth phase C6 cells were digested with $0.25 \%$ trypsin and the cell suspension was collected. The cell viability was more than $95 \%$ and the cell concentration was adjusted to $2.5 \times 106 / \mathrm{ml} .37$ constant temperature shaker stand.

\subsubsection{Preparation before injection}

$12 \mathrm{~h}$ before the rats fasting and water, with $10 \%$ chloral hydrate $(3.5 \mathrm{mg} \cdot \mathrm{kg}-1$ after intraperitoneal injection of anesthesia, cut the top of the head hair $1.5 \mathrm{~cm} \times 1.5 \mathrm{~cm}$, stereotactic instrument fixed, conventional disinfection, along the canthus After the middle point of the line to cut the scalp $1 \mathrm{~cm}$, exposed skull, in the anterior fontanel coronal suture and sagittal suture $1.0 \mathrm{~mm}$, right $3.0 \mathrm{~mm}$ with a triangular needle drill diameter of $1.0 \mathrm{~mm}$ holes, cannot hurt the dura mater.

\subsubsection{Injection cells}

Micro-syringe extraction $25 \mu \mathrm{L} \mathrm{C6}$ cell suspension, the needle is adjusted to touch the dura mater, along the vertical hole into the needle $5.5 \mathrm{~mm}$, back $1 \mathrm{~mm}$, slowly the cell suspension into the caudate nucleus, the injection time is $10 \mathrm{~min}$, after the injection needle 10min after the slow needle. With sterile bone wax closed bone hole, saline irrigation field, spray the right amount of antibiotics, suture the skin, incision sterilization, conventional feeding.

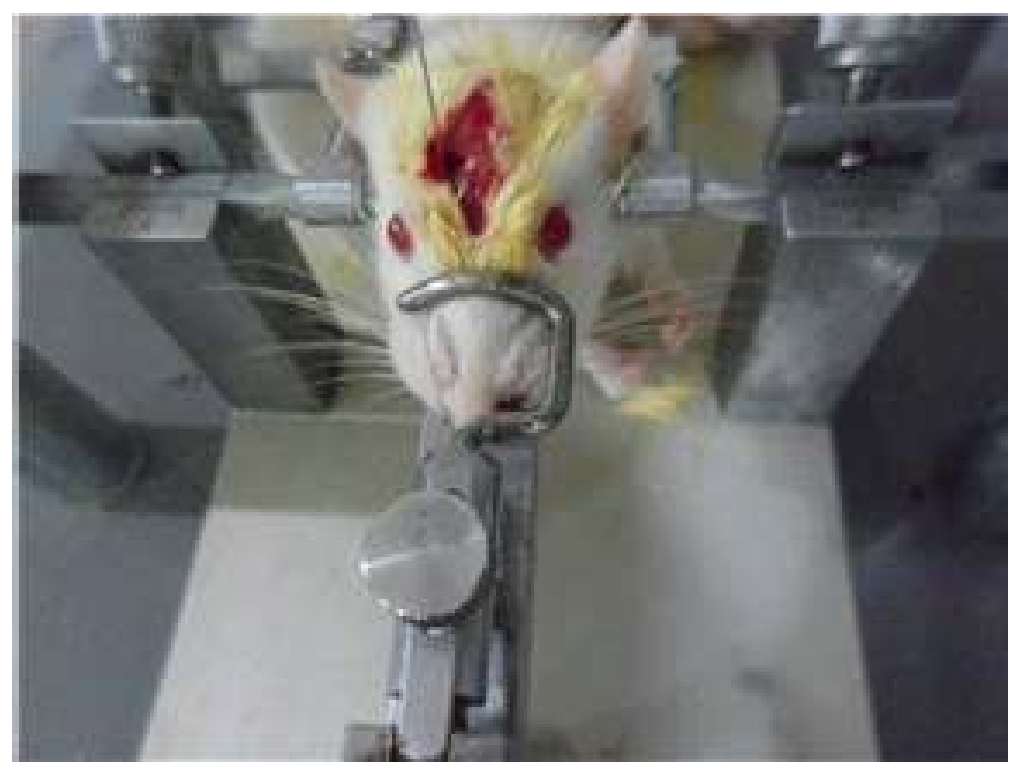

Figure 1. Construction of C6 glioma model in rat brain 


\subsection{Postoperative indicators of detection}

\subsubsection{Observation of rat survival status}

The changes of behavior, body weight change and survival time were observed and recorded.

\subsubsection{MRI examination}

Three rats were randomly selected for 3 weeks, $10 \%$ chloral hydrate was injected intraperitoneally and fixed on the specific coil of rats. MRI scan and enhanced scan were performed with American GE 3.0 NMR.

\subsubsection{Pathological anatomy examination}

After the MRI examination with an excessive amount of $10 \%$ chloral hydrate by intraperitoneal injection of the week group of rats, with saline and 4\% paraformaldehyde was perfused by the left ventricle, and decapitated brain, through the needle crown coronal tumor and Brain tissue, observe the general morphology.

\subsubsection{Histological examination}

HE staining routine pathology was performed. GFAP immunohistochemical examination was performed. Each batch of samples was set up yin and yang control, negative control to PBS instead of primary antibody, positive by the reagent provided by the company. Cytoplasm appears brown or brown as positive cells.

\section{Results}

\subsection{Postoperative survival of rats}

19 weeks after the first day of the resumption of diet, no obvious abnormalities; 2 weeks later, 18 only apathetic, decreased activity, loss of appetite, uneven fur and dull, weight loss, and conjunctival hyperemia, facial unclean. The right side of the limb paralysis and other performance; 3 weeks later, continue to survive in 9 rats, 8 symptoms further increased, periorbital and nasal cavity around severe congestion, limb convulsions, sluggish, significantly reduced diet, weight decreased significantly, $47 \mathrm{~d}$ died; 1 survived $60 \mathrm{~d}$ still no obvious behavior changes and weight loss, autopsy no tumor growth. The survival time of the tumor-bearing rats was $(32.78 \pm 2.34) \mathrm{d}$.

\subsection{MR imaging changes}

10 rats can be seen in the left caudate nucleus have a new biological generation, T1 was slightly lower signal or other signals, T2 image was high signal or mixed signal; enhanced visible new organisms were enhanced, the new creatures have liquefaction, necrosis; new biology diameter of about 6-9 $\mathrm{mm}$, the boundary is clear, visible edema around (Figure 1).

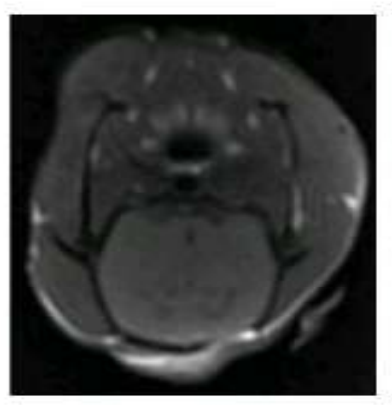

T1

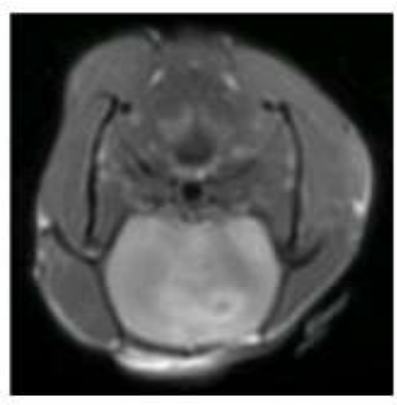

T2

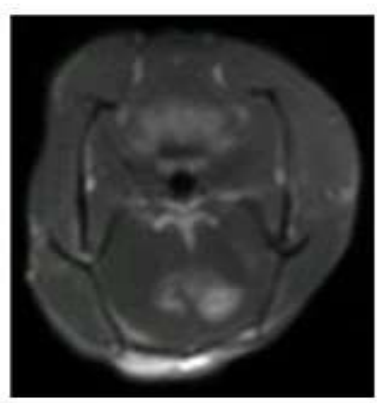

Image enhancement scan

Figure 2. MRI of the head of the tumor-bearing rats after 3 weeks of C6 glioma cell inoculation

\subsection{Pathologic anatomy}

The intracranial tumor boundary was relatively clear, the brain tissue around the tumor was swollen, and the midline was significantly displaced to the right (Figure 2a). 


\subsection{Histological examination results}

\subsubsection{HE staining}

The results of HE staining showed that the tumor cells were confused and dense, and the mitotic images were easy to see. There were vascular tissues in the tumor. The invasive growth of the tumor was finger-like into the surrounding normal tissues (Fig. 2b, c, d).

\subsubsection{Immunohistochemical staining}

GFAP immunohistochemical staining showed strong positive expression of GFAP (Figure 2e). Autopsy of tumor tissue showed extracranial metastatic performance.
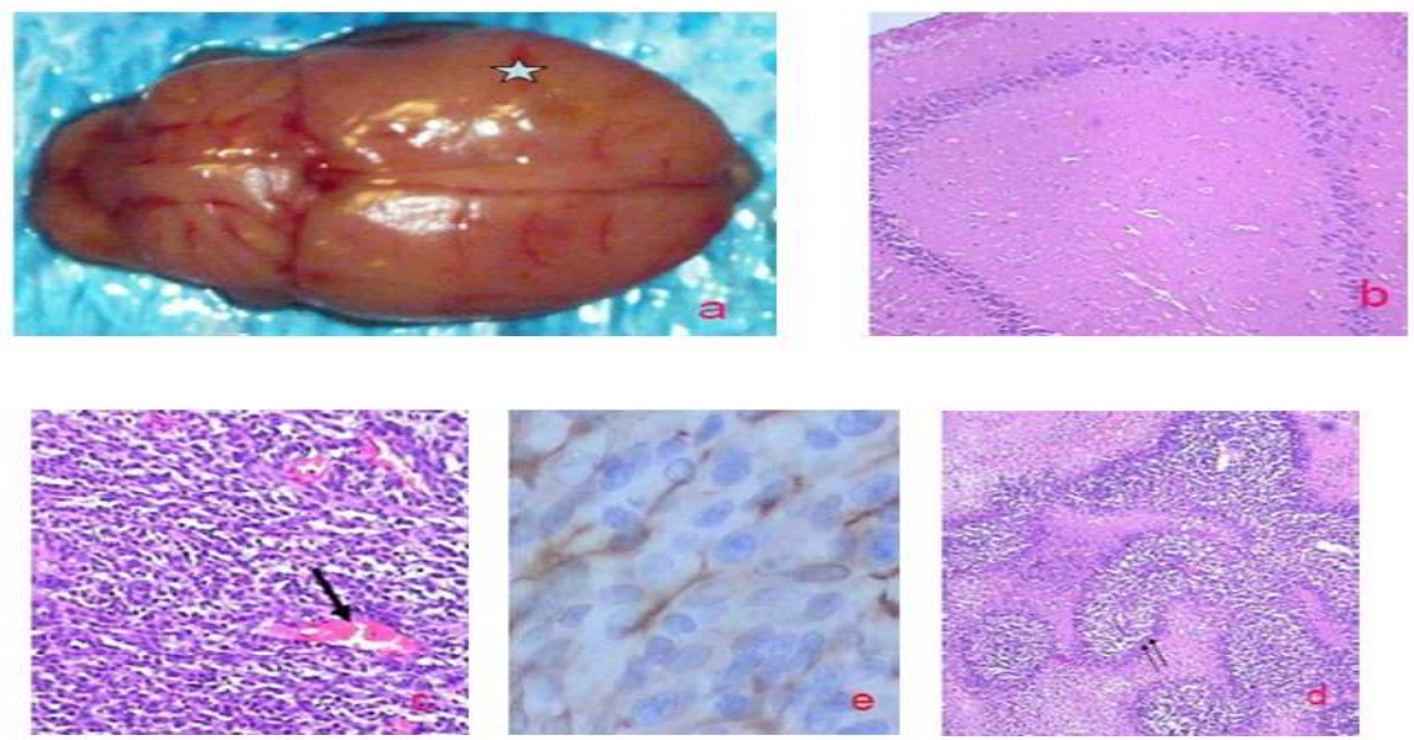

Figure 3. C6 glioma cells inoculated 3 weeks after tumor-bearing rats brain gross and histopathological map

A: Brain tissue specimens, visible tumor (show), the left hemisphere was swollen, midline to the right side of the shift;

B: $\mathrm{N}$ brain tissue $(\mathrm{HE}, \times 100)$, cortical neurons can be seen neatly arranged rules;

$(\mathrm{HE}, \times 200)$, showing cell disordered cells, irregular cell morphology, there are more mitotic images, visible tumor neovascularization ( $\uparrow$ show), which is still visible red blood cells;

$(\mathrm{HE}, \times 100)$. The tumor tissue was infiltrated and the tumor tissue was protruding into the normal tissue $(\uparrow \rightarrow)$. There was no obvious capsule around the tumor.

E: Tumor tissue GAFP immunohistochemical staining $(\mathrm{HE}, \times 400)$, GFAP positive expression was brown.

\section{Discussion}

In this study, based on the literature on the model of orthotopic transplanted glioma in vivo, the relationship between cell number and cell viability, target location, needle depth, cell injection rate, cell and target brain tissue contact time and other aspects of optimization, and achieved satisfactory results.

\subsection{Cell number and cell viability}

First, the viability of C6 glioma cells to be inoculated should be $>90 \%$. If the cell activity is poor, the cells are discarded and cultured again until the test activity is consistent with the modeling needs. Before cell administration, 37 constant temperature shaker, as far as possible in a short time (not more than 90min) inoculation, and gently shaking the cell suspension before each vaccination to prevent cell precipitation lead to cell inoculation uneven; cell number is the impact of the success rate of the model In this study, $1 \times 106 \cdot 10 \mu \mathrm{L}-1,2.5 \times 106 \cdot 25 \mu \mathrm{L}-1,4 \times 106 \cdot 25 \mu \mathrm{L}-1$, $6 \times 106 \cdot \mu \mathrm{L}-1$ vaccination group was observed and found to be the most suitable C6 rat brain glioma cell suspension inoculation volume of $25 \mu \mathrm{L}$, the number of cells is $2.5 \times 106$, the number of cells is too small model success rate is low, 
too high to ensure that the experimental animal modeling success rate but the animal survival status is poor and short survival time, not for further experimental study.

\subsection{Target positioning}

Location of the right mouse caudate putamen was selected as the injection point, and the animal stereotactic instrument was used in the coronal suture $1 \mathrm{~mm}$, the sagittal right $3 \mathrm{~mm}$ into the needle, the depth of about $5 \mathrm{~mm}$ from the dura mater.

\subsection{Needle depth and cell injection rate}

C6 cells along the needle inoculation to the extracranial growth of the tumor model is the main shortcomings, this experiment selected in the brain after the needle back method, that is, advanced needle $5.5-6 \mathrm{~mm}$, and then put $1 \mathrm{~mm}$, resulting in a target location So that the injection process should be slow, if too soon cause the cell suspension from the brain spill, spread along the cerebrospinal fluid, resulting in reduced number of cells, the tumor is difficult to grow into solid tumors, or the cells grow under the scalp and the mold fails.

\section{4. cells and target brain tissue contact time}

This experiment uses a micro-syringe precision draw $25 \mu \mathrm{L}, 10 \mathrm{~min}$ uniform injection is completed, and then in situ needle $10 \mathrm{~min}$. At this time to extend the retention time on the one hand is conducive to the deposition of tumor cells in the bottom of the puncture, reducing the siphon effect caused by the cells along the needle back out of the brain. On the other hand, the brain tissue to adapt to local pressure, reduce tumor cells along the needle planting transfer. Finally, after slow down the needle with bone wax closed skull drilling, can better prevent further inoculation of cells inoculated, and in the wound at the injection of appropriate antibiotics to prevent postoperative infection.

\subsection{Conclusion}

In general, treatment interventions during a slower tumor growth can better determine the therapeutic effect. According to the survival status of the animal model and the pathological findings of the tumor, it can be seen that the tumor in the brain of 7 days or so has existed, but its survival condition is good; $7-14 \mathrm{~g} \cdot \mathrm{d}-1$ rat brain tumor growth, loss of appetite, weight Began to decline; 21d or so occupy the majority of brain tissue in rats, rats hemiplegia, orbital congestion, apathetic, weight to $8-10 \mathrm{~g}$ rate of decline. Based on the above factors, further drug intervention study to select glioma cells after vaccination $10-14 \mathrm{~d}$ is appropriate.

In conclusion, we established the gross glioma tumor model according to the above method, the tumor growth rate was stable, the degree of differentiation was low, the microvessel was rich and the growth was rapid, similar to the growth characteristics of human glioma, Cerebral glioma etiology and pharmacodynamics study of animal models.

\section{References}

1. Ji X, et al. Observation on the growth of intracranial inoculation of stereotactic rat gliomas. Journal of Neurology and Neurology, 2005,12 (5): 311-314.

2. Wu J, et al. Establishment of rat C6 glioma model and observation of intracranial tumor growth [J]. Journal of the Fourth Military Medical University, 2000,21 (3): 307-310.

3. Fang S, et al. Study on apoptosis of C6 glioma cells induced by local thermochemotherapy [J]. Chinese Journal of Clinical Neurosurgery, 2003, 8 (2): 127-130.

4. Zhu H, et al. Study on animal model of glioma and its application [J]. Zoological Research 2012, Jun.33 (3): 337-342.

5. Qiu D, et al. Effects of indwelling time after injection on C6 rat glioma model [J]. Journal of Chongqing Medical University, 2008,33 (10): 1260- 1263.

6. Bai Y, et al. Establishment of rat brain glioma animal model and its morphological observation [J]. Journal of Xuzhou Medical College, 2004,24 (4): 331- 334.

7. Watanabe K, et al. Feasibility and limitations of the rat model by C6 gliomas implanted at the subcutaneous region [J]. Neurol Res, 2002, 24: 485-490.

8. Del Duca D, et al. Spheroid preparation from hanging drops: characterization of a model of brain tumor invasion [J]. $\mathrm{J}$ Neurooncol, 2004, 67: 295-330.

9. Kondziolka D, et al. Radiobiology of radiosurgerg: Part. The rat C6 glioma model [J]. Neurosurgery, 2007, 31 (2): $280-287$.

10. Kinoshita N, et al. Usefulness of basic fibroblast growth factor for radiation-exposed tissue [J]. Wound Repair Regen, 2012,20 (1): 91-102. 